\title{
Multi-drug resistance of blood stream, urinary tract and surgical site nosocomial infections of Acinetobacter baumannii and Pseudomonas aeruginosa among patients hospitalized at Felegehiwot referral hospital, Northwest Ethiopia: a cross- sectional study
}

Hilina Motbainor, Fetlework Bereded and Wondemagegn Mulu*

\begin{abstract}
Background: Multi-drug resistant (MDR) Acinetobacter baumannii and Pseudomonas aeruginosa are major causes of nosocomial infections globally. They are the current World Health Organization critical priority pathogens for resistance, Antimicrobial resistance (AMR) surveillance and discovery of new antibiotics. However, there is paucity of data on nosocomial infections (NIs) caused by such superbugs in Ethiopia. Therefore, this study determined the magnitude and profile of nosocomial MDR A. baumannii and P. aeruginosa infections among patients hospitalized at Felegehiwot referral hospital, Northwest Ethiopia.

Methods: A cross-sectional study was conducted at Felegehiwot referral hospital from April 1 to July 31, 2018. A total of 238 patients with blood stream, urinary tract and surgical site Nls were enrolled conveniently. Either blood, urine and wound swab specimens were collected and processed using standard bacteriological procedures. A. baumannii and $P$. aeruginosa isolates were identified using standard bacteriological techniques and confirmed by automated Vitek2 Compact. Antimicrobial susceptibility testing on isolates was performed using the disk diffusion technique. The results were interpreted as per the standard zone sizes of Clinical and Laboratory Standards Institute.Chi-square test was done to determine associations among variables. $P$ value $<0.05$ was considered statistical significant.

* Correspondence: wondem_32@yahoo.com

Department of Medical Laboratory Science, College of Medicine and Health

Sciences, Bahir Dar University, Bahir Dar, Ethiopia

(c) The Author(s). 2020 Open Access This article is distributed under the terms of the Creative Commons Attribution 4.0 International License (http://creativecommons.org/licenses/by/4.0/), which permits unrestricted use, distribution, and reproduction in any medium, provided you give appropriate credit to the original author(s) and the source, provide a link to the Creative Commons license, and indicate if changes were made. The Creative Commons Public Domain Dedication waiver (http://creativecommons.org/publicdomain/zero/1.0/) applies to the data made available in this article, unless otherwise stated. 
(Continued from previous page)

Results: The median age of participants was 29 years. Overall,20(8.4\%) of patients had nosocomial MDR A. baumannii and $P$. aeruginosa infections. The proportion of nosocomial MDR blood stream, urinary tract and surgical site infections were 13(8.9\%), 5(8.3\%) and 2 (6.3\%), respectively. Patients with NI had lower mean age (24.9 years) $(P=0.035)$. All isolates of Nls were from patients with intravenous catheterization. The frequency of $\mathrm{NI}$ was $9(3.8 \%)$ for MDR A. baumannii and 11(4.6\%) for MDR P.aeruginosa. A. baumannii and P. aeruginosa isolates were 100\% MDR. All isolates of $A$. baumannii and $P$. aeruginosa were $100 \%$ resistant to ampicillin and piperacillin. $A$. baumannii isolates were 33.3 and $44.5 \%$ resistance against meropenem and ciprofloxacin, respectively while P.aeruginosa isolates revealed 36.4 and $45.5 \%$ resistance against ciprofloxacin and meropenem, respectively.

Conclusions: Health care associated infections of MDR A.baumannii and P. aeruginosa are critical problems in the study area. Therefore, urgent focused interventions required to contain the spreading of MDR NIs. Treatment of NIs for patients on health care should be guided by antimicrobial susceptibility testing.

Keywords: NIs, MDR, A. baumannii, P. aeruginosa, Ethiopia

\section{Background}

Multidrug-resistant (MDR) gram-negative bacterial infections are recognized as one of the major threats to global health. They are leading causes of nosocomial infections in the world [1-3]. NI is an infection occurring in a patient at the time of care in a hospital that was not manifest or incubating during admission but developed after $48 \mathrm{~h}$ of hospitalization [4].

The hospital environment contains a large number of immuno-compromised individual's and patients with diverse bacterial pathogens and normal microbiota $[1,5]$. NI accounted 7-10\% prevalence in the world [5]. According to 2014 World Health Organization (WHO) report, $15 \%$ of all the hospitalized patients suffered from NIs [6].

Surgical site, blood stream and urinary tract infections are the most frequently reported types of NIs [1, 7]. Urinary tract infection (UTI) which involves the bladder, ureter and kidney is the most common type of NIs accounting, 31\% [8]. UTI caused by A. baumannii and $P$. aeruginosa are usually hospital acquired and related to urinary tract catheterization or surgery $[9,10]$. Surgical site infection (SSI) can involve the skin, subcutaneous tissue of the incisions, deep soft tissues of the incisions and any part of organ and spaces and accounted for $17 \%$ of NIs $[8,10]$. Blood stream infection is also one of the common NIs next to UTI and SSIs [10]. It can be acquired from catheter, secondary to another infection site, invasive diagnostic procedures or foreign body [10].

Nosocomial infections with MDR organisms are major global health issues [1-3]. They are very difficult for treatment and main causes of poor clinical outcome, morbidity, mortality, prolonged hospitalization and high health care costs $[11,12]$. The situation is true and urgent in Ethiopia.

The non-fermentative gram negative bacilli $A$. baumannii and $P$. aeruginosa have emerged as serious particular concern $[13,14]$. They are among the most common and serious MDR pathogens documented along with Enterococcus faecium, Staphylococcus aureus, Klebsiella pneumoniae and Enterobacter spp., collectively acronyms and considered as ESKAPE pathogens and superbugs, respectively $[14,15]$.

Multi-drug resistant $A$. baumannii and $P$. aeruginosa survive in the hospital setting, transmitted easily between patients through the hands of health care workers $[2,14]$. Earlier findings elsewhere in the world reported that $A$. baumannii and $P$. aeruginosa commonly possess inherent resistance to antimicrobial agents through reduced permeability of the outer membrane, efflux pump systems, enzymatic inactivation and biofilm formation $[15,16]$. Thus, they are often resistant to almost all $\beta$ lactams, aminoglycosides and quinolones [15, 17].

Nosocomial isolates of MDR A. baumannii and P. aeruginosa complicated the treatment of infections and had adverse effect on clinical outcomes and increases patient treatment costs [18]. Factors such as antimicrobial drug overuse, prescription of drugs without susceptibility testing, self- medication and long duration of hospitalization are reported to the occurrence of $\operatorname{MDR}[11,19]$. However, there is a scarcity of data on the burden of nosocomial MDR A. baumannii and P. aeruginosa infections in Ethiopia in general and study area in particular. Unavailability of local antibiogram data linked with self-drug prescription by patients and poor awareness on antimicrobial resistance are also big issues. Thus, the study aimed at determining the proportion of nosocomial MDR A. baumannii and $P$. aeruginosa infection among patients clinically diagnosed for NIs at Felegehiwot referral Hospital (FHRH), Ethiopia.

\section{Methods}

Study design, period and setting

A cross-sectional study was conducted from April 1 to July 31, 2018 at FHRH, Bahir Dar, Northwest Ethiopia. Bahir Dar is a capital city of the Amhara National 
Regional State, located approximately $565 \mathrm{~km}$ northwest of Addis Ababa. FHRH is a tertiary hospital that provides services for 5 to 7 million people. It has 684 health care and 166 administrative workers. The hospital has 466 beds for inpatient services. FHRH consists of 13 different wards including Surgical, Medical, Gynaecology and Intensive Care Units (ICUs). It has adult and neonatal ICUs with 8 beds. Bacteriological procedures were conducted at FHRH microbiology laboratory and further confirmatory identification of isolates and screening of MDR made using VITEK2 compact at Amhara Public Health Institute (APHI), Ethiopia. All patients admitted at FHRH and clinically presumptive for either of nosocomial urinary tract, post-operative surgical site and blood stream infection during the study period were the study population.

\section{Sample size and sampling}

The sample size for NIs was determined using Epi info version 3.5.1 (public domain software, www.cdc.gov) by considering 95\% confidence level and marginal error (5\%). A proportion of 0.83 MDR NIs taken from previous study in other parts of South East Ethiopia [16]. Thus, the total sample size was 238. All patients who were clinically presumptive for nosocomial blood stream, urinary tract and post-operative surgical site nosocomial infections were included conveniently until the required sample size was achieved.

\section{Variables}

Multi-drug resistant blood stream, urinary tract and surgical site nosocomial infections $A$. baumannii and $P$. aeruginosa were the dependent variables while demographic variables (age, sex, residence, educational status, occupation) and clinical data on co-morbidity, urinary catheterization, intravenous catheterization, duration of catheterization, previous history of antibiotics, previous history of surgery, duration of hospitalization, wards of patients hospital and duration of operation were the independent variables.

\section{Inclusion and exclusion criteria}

All patients who had either clean and cleancontaminated operations or other medical reasons admitted in Medical, Surgical and ICU wards of FHRH and developed clinical evidences of blood stream, urinary tract and surgical site nosocomial infections after $48 \mathrm{~h}$ of admission were included during the study period. However, patients who had re-operation, contaminated and dirty operations, ventilator- associated pneumonia, skin infections and developed any type of clinical evidences of infection within $48 \mathrm{~h}$ of admission were excluded from the study.

\section{Data collection}

Information on demographic variables was collected from each participant by face-to-face interview using a structured questionnaire. Clinical data related to comorbidities, hospitalization, surgery and use of antibiotics were collected by reviewing patient's medical record and in consultation with the respective physician. With the study team, patients admitted in the Medical, Surgical and ICU wards were carefully diagnosed for NIs by Internists and Surgeon. Clinical specimens (blood, urine and wound swab) were collected by the study team as soon as NI was reported following the bacteriological standard procedures.

The criteria of The European Centre for Disease Prevention and Control were used to define NIs [10]. Patients who had either purulent drainage, pain, localized swelling, redness, or heat in the skin, subcutaneous tissue, deep soft tissue, organ or spaces and one positive culture for A. baumannii and P. aeruginosa after $48 \mathrm{~h}$ of operation were considered as nosocomial surgical site infection. Patients who had either fever $\left(>38^{\circ} \mathrm{C}\right)$, urgency, frequency, dysuria, or suprapubic tenderness with no other recognized cause but has $\geq 10^{2} \mathrm{CFU} /$ milliliters $(\mathrm{ml})$ and $\geq 105 \mathrm{CFU} / \mathrm{ml}$ of urine culture for catheterized and non- catheterized patients, respectively after $48 \mathrm{~h}$ of admission were considered as nosocomial urinary tract infection. On the other hand, patients who had either fever $\left(>38^{\circ} \mathrm{C}\right)$, chills, or hypotension and one positive blood culture for $A$. baumannii and $P$. aeruginosa after $48 \mathrm{~h}$ of admission were considered as nosocomial blood stream infection.

\section{Wound swab collection and processing}

Two wound samples from each participant were collected aseptically by sterile cotton swabs dipped in normal saline using Levine method [20]. The wound swabs were inoculated on MacConkey agar (MAC) and Blood agar (BA) (Oxoid, UK) at a time. Both plates were incubated at $37^{\circ} \mathrm{C}$ and examined for visible bacterial growth after $48 \mathrm{~h}$ of incubation.

\section{Blood sample collection and processing}

As per the standard protocol, 1, 2 and $10 \mathrm{ml}$ of venous blood were collected from neonates, children and adults presumptive for blood stream infection, respectively [21]. Following cleaning of the site of blood collection with $70 \%$ alcohol and 2\% tincture iodine, two blood samples were collected from two different sites of peripheral vein of each febrile patient using two bottles of blood sample within $30 \mathrm{~min}$ difference. The collected blood samples were inoculated directly to $5-10 \mathrm{ml}$ Tryptic Soya broth blood culture medium bottle (Oxoid, UK) and transported to FHRH microbiology laboratory. All blood culture broths were incubated aerobically at 
$37^{\circ} \mathrm{C}$ and regular subcultures were done on $\mathrm{BA}$ and MAC (Oxoid, UK) after 1, 2 and then 3 days later daily up to 7 days of incubation. All of the inoculated agar plates were incubated at $37^{\circ} \mathrm{c}$. Finally, plates were examined for bacterial growth after $24 \mathrm{~h}$.

\section{Urine sample collection and processing}

Clean-catch mid-stream urine was collected from patients presumptive for UTI. From catheterized patients, $5 \mathrm{ml}$ of catheterized urine transferred to a sterile container after cleansing the out let of catheter. For noncatheterized patients, the same amount of urine sample was collected by the patient using sterile container. Urine samples were inoculated on MAC and BA (Oxoid, UK). All agar plates were incubated aerobically at $37^{\circ} \mathrm{C}$ for $24 \mathrm{~h}$ and observed for bacterial growth. Blood agar colonies were counted using colony counter and checked for significant bacteriuria. Culture from catheterized and non-catheterized patients that grew $\geq 10^{2}$ $\mathrm{CFU} / \mathrm{ml}$ and $10^{5} \mathrm{CFU} / \mathrm{ml}$, respectively was taken as a significant bacteriuria, respectively. For heterogeneous colonies, sub-culturing of individual distinct colonies was performed to ensure pure cultures.

\section{Identification of bacterial isolates}

All isolates of $A$. baumannii and $P$. aeruginosa were identified by manual standard microbiological methods [21]. All A.baumannii and P. aeruginosa suspected isolates were also further confirmed by an automated Vitek2 Compact (BioMérieux, France).

\section{Antimicrobial susceptibility testing}

Antimicrobial susceptibility testing was carried out for each isolates of $A$. baumannii and $P$. aeruginosa on Mueller Hinton agar (MHA) (Oxoid, UK) by Kirby Bauer disk diffusion technique [22]. All A. baumannii and $P$. aeruginosa isolates were tested against the following classes of antibiotics: Penicillin (ampicillin $(10 \mu \mathrm{g})$ ), piperacillin $(100 \mu \mathrm{g})), \quad \beta$-lactam/ $\beta$-lactamase inhibitor combination (amoxicillin-clavulanic acid $(20 / 10 \mu \mathrm{g})$ ), cephalosporin (ceftazidime $(30 \mu \mathrm{g})$, cefotaxime $(30 \mu \mathrm{g})$ and ceftriaxone $(30 \mu \mathrm{g})$ ), aminoglycosides (gentamycin $(10 \mu \mathrm{g}))$, fluoroquinolones (ciprofloxacin $(10 \mu \mathrm{g})$ ), Folate pathway inhibitor (trimethoprim-sulphamethoxazole $(1.25 / 23.75 \mu \mathrm{g}))$, carbapenem (meropenem $(10 \mu \mathrm{g})$ ) and tetracycline $(30 \mu \mathrm{g})$ (Oxoid, England). The results were interpreted using the standard zone sizes of the Clinical and Laboratory Standards Institute (CLSI, 2017) guidelines [23].

\section{Quality control}

The prepared questionnaire was checked for its completeness and validity prior to the data collection. All the standard operating procedures (SOPs) were strictly followed at all stages of microbiological analysis. American Type Culture Collection (ATCC) standard reference strains ( $P$. aeruginosa ATCC27853 and E. coli ATCC 25922) were used for quality control of antimicrobial susceptibility testing. MDR $A$. baumannii and $P$. aeruginosa isolates were confirmed by using Vitek2 compact. A standardized bacteriological procedure was followed to maintain correct laboratory results. At regular intervals and whenever a new batch of strain or reagent is prepared, standard strains of $P$. aeruginosa ATCC27853 and E. coli ATCC 25922 were used as positive controls. The sterility of the media was checked by incubating the media overnight before its use. The data were checked for completeness and representativeness prior to entry.

\section{Data analysis}

Data were checked, entered and analyzed using Statistical Package for Social Science 23 (IBM Corp Released 2011.IBM SPSS statistics. Armonk, NY: IBM Corp). Descriptive statistics were used to describe relevant variables. Chi-square test, Fishers exact test and Independent samples $\mathrm{T}$ Test was obtained to determine association between dependent and independent variables. $P$ value of $<0.05$ was considered statistical significant.

\section{Results}

\section{Demographic characteristics}

A total of 238 patients with clinical evidence of nosocomial infection (BSI, UTI and SSI) were enrolled in the study. Of them, 129 (54.2\%) were males. The majority (21.4\%) of participants were found in the age group of $>$ 51 years with median age of 29 years. One hundred twenty six (52.9\%) of the study participants were from urban settings. Data on occupation showed that majority (39.1\%) of participants were government employee. Table 1 depicts the demographic characteristics of the study participants.

\section{Rate of nosocomial infection and frequency of bacterial isolates}

The overall prevalence of the combined nosocomial MDR A. baumannii and P. aeruginosa infection was 20 $(8.4 \%)$. Of them, the proportion of BSI, UTI and SSI were $13(8.9 \%), 5(8.3 \%)$ and $2(6.3 \%)$, respectively. The proportion of nosocomial MDR A. baumannii and $P$. aeruginosa infection were $9(3.8 \%)$ and $11(4.6 \%)$, respectively. P. aeruginosa accounted for $6.3,4.8$ and $3.3 \%$ of nosocomial SSI, BSI and UTI, respectively while, MDR A. baumannii causes 5 and $4.1 \%$ of nosocomial UTI and BSI, respectively (Table 2).

Participants with NIs had lower mean of age (24.9 years) than those without NIs (29.6 years) and the difference was statistical significant $(P=0.035)$. Highest 
Table 1 Demographic characteristics of patients clinically presumptive for nosocomial infection at FHRH, $2018(n=238)$

\begin{tabular}{|c|c|c|}
\hline Demographic variables & Number & Percent \\
\hline \multicolumn{3}{|l|}{$\overline{\text { Sex }}$} \\
\hline Female & 109 & 45.8 \\
\hline Male & 129 & 54.2 \\
\hline \multicolumn{3}{|l|}{ Age (in years) } \\
\hline$<1$ & 31 & 13.0 \\
\hline $1-10$ & 34 & 16.4 \\
\hline $11-20$ & 29 & 12.2 \\
\hline $21-30$ & 47 & 19.7 \\
\hline $31-40$ & 25 & 10.5 \\
\hline $41-50$ & 21 & 8.8 \\
\hline$>51$ & 51 & 21.4 \\
\hline \multicolumn{3}{|l|}{ Residence } \\
\hline Urban & 126 & 52.9 \\
\hline Rural & 112 & 47.1 \\
\hline \multicolumn{3}{|l|}{ Occupation } \\
\hline Farmer & 23 & 15.2 \\
\hline House wife & 31 & 20.5 \\
\hline Government employee & 59 & 39.1 \\
\hline Private employee & 27 & 17.9 \\
\hline Other work & 11 & 7.3 \\
\hline Unemployed & 87 & 36.6 \\
\hline \multicolumn{3}{|l|}{ Education } \\
\hline Under school age & 49 & 20.6 \\
\hline Illiterate & 52 & 27.5 \\
\hline Elementary completed & 60 & 31.7 \\
\hline Highschool completed & 11 & 5.8 \\
\hline Diploma & 36 & 19.0 \\
\hline Degree and above & 30 & 15.9 \\
\hline
\end{tabular}

Key: Unemployed: Below school age + students; Other work: daily laborer and merchant

(15.4\%) NIs rate was found in age groups $<10$ years. The proportion of NI was $13(11.4 \%)$ in those participant with co-morbidity. All isolates of NIs were from patients with intravenous catheterization (Table 3). Duration of operation was significantly higher in those patients with

Table 2 Proportion of nosocomial MDR A. baumannii and $P$. aeruginosa infection in patients clinically presumptive for nosocomial blood stream, urinary tract and surgical site infection at FHRH, $2018(n=238)$

\begin{tabular}{lllll}
\hline \multirow{2}{*}{$\begin{array}{l}\text { Type of MDR } \\
\text { isolates }\end{array}$} & \multicolumn{4}{l}{ Rate of nosocomial infection N (\%) } \\
\cline { 2 - 5 } & $\mathrm{UTI}(n=60)$ & $\mathrm{SSI}(n=32)$ & $\mathrm{BSI}(n=146)$ & Total \\
\hline A. baumannii & $3(5)$ & $0(0)$ & $6(4.1)$ & $9(3.8)$ \\
P. aeruginosa & $2(3.3)$ & $2(6.3)$ & $7(4.8)$ & $11(4.6)$ \\
Total & $5(8.3)$ & $2(6.3)$ & $13(8.9)$ & $20(8.4)$ \\
\hline
\end{tabular}

confirmed NIs (180 min) than their counterparts (155 $\min ) \quad(P=0.04)$. Moreover, the duration of catheterization was higher in those with confirmed NIs (13.6 days) than their counter parts (11.3 days). However, the difference was not statistical significant $(P=0.25)$ (Table 3).

\section{Multi-drug resistance profiles of $A$. baumannii and $P$. aeruginosa isolates}

$A$. baumannii and $P$. aeruginosa isolates were resistant to three to six antibiotics from different classes. All isolates of $A$. baumannii (9) and $P$. aeruginosa (11) were MDR (100\%). Among this, 3 (33.3\%) isolates of $A$. baumannii and $4(36.4 \%)$ isolates of $P$. aeruginosa showed resistance to antibiotics from six different classes, respectively. Three (33.3\%) isolates of A. baumannii and 1 (9.1\%) isolates of $P$. aeruginosa were resistant against antibiotics from 4 different classes, respectively. Moreover, 3 (33.3\%) of isolates of A. baumannii and 6 (54.5\%) of $P$. aeruginosa isolates were resistant against antibiotics from three different classes (Table 4).

\section{Antimicrobial resistance profiles of $A$. baumannii and $P$. aeruginosa}

Both A. baumannii and P. aeruginosa isolates showed $100 \%$ resistance against ampicillin and piperacillin. Besides, P. aeruginosa isolates showed 100\% resistance against amoxicillin-clavulanic acid, cefotaxime, ceftriaxone and trimethoprim-sulphamethoxazole. A. baumannii isolates showed resistance $88.9 \%$ to amoxicillin-clavulanic acid, ceftriaxone and cefotaxime and also $77.8 \%$ to tetracycline and ceftazidime. However, $P$. aeruginosa isolates revealed a high resistance rate to ceftazidime $(63.6 \%)$ and tetracycline (90.6\%). A. baumannii isolates showed low level resistance rate to ciprofloxacin (44.5\%) and meropenem (33.3\%). Similarly, P. aeruginosa isolates showed low level of resistance against ciprofloxacin (36.4\%) and meropenem (45.5\%) (Table 5).

\section{Discussion}

Antibiotic resistant nosocomial infections are becoming serious health care problem in ICU and other areas of hospital care, increasing morbidity, mortality, length of stay, and health care costs [2, 24]. The epidemiological and antimicrobial resistance profiles of NIs showed variations among hospitals around the globe. Many of the infections are caused by bacteria that are resistant to multiple antibiotics [24, 25]. This study showed the proportion of NIs due to two MDR non-fermentative gram negative bacilli among patients hospitalized in different wards of a referral hospital.

In the present study, $8.4 \%$ of patients were infected with nosocomial MDR A. baumannii and P. aeruginosa. This indicated that MDR A.baumannii and $P$. aeruginosa 
Table 3 Distribution of nosocomial MDR A. baumannii and $P$. aeruginosa infection in different variables of study participants clinically presumptive for nosocomial infection at FHRH, $2018(n=238)$

\begin{tabular}{|c|c|c|c|c|c|}
\hline \multirow[t]{2}{*}{ Variables } & & \multicolumn{3}{|c|}{ Confirmed rate of Nosocomial infection } & \multirow[t]{2}{*}{$P$-value } \\
\hline & & $\begin{array}{l}\text { Positive } \\
\mathrm{N}(\%)\end{array}$ & $\begin{array}{l}\text { Negative. } \\
\mathrm{N}(\%)\end{array}$ & $\begin{array}{l}\text { Total. } \\
\mathrm{N}(\%) \\
\end{array}$ & \\
\hline \multirow[t]{2}{*}{ Sex } & Female & $9(8.3)$ & $100(91.7)$ & $109(45.8)$ & \\
\hline & Male & $11(8.5)$ & $118(91.5)$ & $129(54.2)$ & 0.94 \\
\hline \multirow[t]{7}{*}{ Age } & $<1$ & $5(16.1)$ & $26(83.9)$ & $31(13.0)$ & \\
\hline & $1-10$ & $5(14.7)$ & $29(85.3)$ & $34(16.4)$ & \\
\hline & $11-20$ & $1(3.4)$ & $28(96.6)$ & $29(12.2)$ & 0.34 \\
\hline & $21-30$ & $2(4.3)$ & $45(95.7)$ & $47(19.7)$ & \\
\hline & $31-40$ & $2(8)$ & $23(92)$ & $25(10.5)$ & \\
\hline & $41-50$ & $0(0)$ & $21(100)$ & $21(8.8)$ & \\
\hline & $>51$ & $5(9.8)$ & $46(90.2)$ & $51(21.4)$ & \\
\hline \multirow[t]{2}{*}{ Residence } & Urban & $9(7.1)$ & $117(92.9)$ & $126(52.9)$ & \\
\hline & Rural & $11(9.8)$ & $101(90.2)$ & $112(47.1)$ & 0.46 \\
\hline \multirow[t]{3}{*}{ Education } & Non-educated & $3(5.8)$ & $49(94.2)$ & $52(27.5)$ & \\
\hline & Educated & $8(5.8)$ & $129(94.2)$ & $137(31.7)$ & \\
\hline & Below school age & $9(18.4)$ & 40 (81.6) & 49 (20.6) & 1.00 \\
\hline \multirow[t]{3}{*}{ Type of NI } & UTI & $5(8.3)$ & $55(91.7)$ & $60(25.2)$ & \\
\hline & SSI & $2(6.3)$ & $30(93.8)$ & $32(13.4)$ & \\
\hline & BSI & $13(8.9)$ & $133(91.1)$ & $146(61.3)$ & \\
\hline \multirow[t]{6}{*}{ Occupation } & Farmer & $0(0)$ & $23(100)$ & $23(15.2)$ & \\
\hline & House wife & $4(12.9)$ & $27(87.1)$ & $31(20.5)$ & 0.20 \\
\hline & Government employee & $5(8.5)$ & $54(91.5)$ & $59(39.1)$ & \\
\hline & Private employee & $0(0)$ & $27(100)$ & $27(17.9)$ & \\
\hline & Other & $1(9.1)$ & $10(90.9)$ & $11(7.3)$ & \\
\hline & Unemployed & $10(11.5)$ & 77 (88.5) & 87 (36.6) & \\
\hline \multirow[t]{3}{*}{ Ward of patients hospital } & Surgical & $4(8.5)$ & $43(91.5)$ & $47(19.7)$ & \\
\hline & Medical & $11(8.1)$ & $125(91.9)$ & $136(80.3)$ & \\
\hline & $\mathrm{ICU}$ & $5(9.1)$ & $50(90.9)$ & $55(23.1)$ & 0.48 \\
\hline \multirow[t]{2}{*}{ Underlying disease } & Yes & $13(11.4)$ & $101(88.6)$ & $114(47.9)$ & 0.11 \\
\hline & No & $7(5.6)$ & $117(94.4)$ & $124(52.1)$ & \\
\hline \multirow[t]{2}{*}{ Urinary Catheterization } & Yes & $9(7.4)$ & $112(92.6)$ & $121(50.8)$ & \\
\hline & No & $11(9.4)$ & $106(90.6)$ & $117(49.2)$ & 0.59 \\
\hline \multirow[t]{2}{*}{ Intravenous Catheterization } & Yes & $20(9.0)$ & $201(91.0)$ & $221(92.9)$ & 0.37 \\
\hline & No & $0(0)$ & $17(100)$ & $17(7.1)$ & \\
\hline \multirow[t]{2}{*}{ Previous antibiotics } & Yes & $14(9.0)$ & $141(91.0)$ & $155(65.1)$ & 0.633 \\
\hline & No & $6(7.2)$ & $77(92.8)$ & $83(34.9)$ & \\
\hline \multirow[t]{2}{*}{ Previous surgery } & Yes & $5(10.2)$ & $44(89.8)$ & 49 (20.6) & 0.61 \\
\hline & No & $15(7.9)$ & $174(92.1)$ & $189(79.4)$ & \\
\hline \multicolumn{2}{|l|}{ Total } & $20(8.4)$ & 218 (91.6) & $238(100)$ & \\
\hline \multicolumn{2}{|l|}{ Mean Age of participants } & 24.9 & 29.6 & & 0.035 \\
\hline \multicolumn{2}{|c|}{ Duration of hospitalization (days) } & 14.5 & 15.7 & & 0.48 \\
\hline \multicolumn{2}{|c|}{ Duration of operation (Minutes) } & 180 & 155 & & 0.04 \\
\hline \multicolumn{2}{|c|}{ Duration of catheterization (Days) } & 13.6 & 11.3 & & 0.25 \\
\hline
\end{tabular}


Table 4 Multi-drug resistance profile of A. baumannii and $P$. aeruginosa isolates from patients clinically presumptive for nosocomial infection at $\mathrm{FHRH}, 2018(n=20)$

\begin{tabular}{|c|c|c|c|c|}
\hline Bacterial isolate & Antibiogram Profile & Antibiotic Class & $\begin{array}{l}\text { Frequency } \\
\mathrm{N}(\%)\end{array}$ & Over all, MDR \\
\hline \multirow[t]{6}{*}{ A. baumannii $(n=9)$} & (AMP,AMC,CAZ,PIP,CIP,CN,SXT,MEM) & 6 & $3(33.3)$ & \\
\hline & (AMP,AMC,CAZ,PIP,CN,SXT) & 4 & $2(22.2)$ & \\
\hline & (AMP,AMC,CAZ,PIP,CIP,CN) & 4 & $1(11.1)$ & \\
\hline & $(\mathrm{AMP}, \mathrm{AMC}, \mathrm{PIP}, \mathrm{CN}, \mathrm{SXT})$ & 3 & $2(22.2)$ & \\
\hline & $(\mathrm{AMP}, \mathrm{CAZ}, \mathrm{PIP}, \mathrm{SXT})$ & 3 & $1(11.1)$ & \\
\hline & & & & $9(100)$ \\
\hline \multirow[t]{4}{*}{ P. aeruginosa $(n=11)$} & (AMP,AMC,CAZ,PIP,CIP,CN,SXT,MEM) & 6 & $4(36.4)$ & \\
\hline & (AMP,AMC,CAZ,PIP,CIP,SXT) & 4 & $1(91)$. & \\
\hline & $(\mathrm{AMP}, \mathrm{AMC}, \mathrm{PIP}, \mathrm{CN}, \mathrm{S} X \mathrm{~T})$ & 3 & $2(18.2)$ & \\
\hline & (AMP,AMC,CAZ,PIP,SXT) & 3 & $4(36.4)$ & \\
\hline
\end{tabular}

$11(100)$

Key: AMP: Ampicillin, AMC: Amoxicillin-clavulanic acid, PIP: Piperacillin, CAZ: Ceftazidime, CIP: Ciprofloxacin, CN: Gentamicin, MEM: Meropenem and SXT:

Sulphamethoxazole- Trimethoprim, R3,4,6, resistance to 3,4,6 antibiotic drug classes

infections are the major health problem in the clinical area in Ethiopia. High patient load, overcrowding, poor infrastructure, poor infection control practices of the hospital and differences in trained medical staff for aseptic procedures might be the possible explanations. This finding was coherent with reports in Tikur Anbessa Hospital, Ethiopia (8.12\%) [25], Uganda (7.39\%) [26], Italy (9.3\%) [27] and Gaza city (6.9\%) [28]. However, it was higher compared to reports from Hiwot Fana Hospital, Ethiopia (0.5\%) [8], Gabon (5.7\%) [29], China (0.78\%) [30] and Indonesia (3.5\%) [31]. In contrast, the overall nosocomial MDR $A$. baumannii and $P$. aeruginosa infections in the present study was lower than studies done in Ghana (23.5\%) [32]. This might be due to variation in sample size, clinical site of infection, age of patients, hospital setting, duration of hospitalization, patients exposure to high risk devices or surgical procedures, microbiological methods employed for screening of MDR resistant strains.

The highest proportion of MDR non-fermentative gram negative bacilli NI infection among the lower age groups in the present study is consistent with earlier studies elsewhere in the world [29, 33, 34]. On the other hand, in the present study all NIs observed among patients with intravenous catheterization. The rate of nosocomial infections was also significantly higher among patients who had prolonged time of operation than their counter parts. This was consistent with previous study in Tikur Anbessa Hospital, Ethiopia (25). This might be due to the high rate of exposure of patients to the two MDR pathogens from the hospital environment, health

Table 5 Antimicrobial resistance profiles of A. baumannii and $P$. aeruginosa isolates from participants presumptive for nosocomial infection at FHRH, Bahir Dar, Northwest Ethiopia, April to July, 2018

\begin{tabular}{|c|c|c|c|c|c|c|}
\hline \multirow[t]{2}{*}{ Antimicrobials } & \multicolumn{2}{|c|}{ A.baumannii } & \multicolumn{2}{|c|}{ P.aeruginosa } & \multicolumn{2}{|c|}{ Total } \\
\hline & $\# T$ & $\mathrm{R} \%$ & $\# \mathrm{~T}$ & $\mathrm{R} \%$ & $\# T$ & $\mathrm{R} \%$ \\
\hline Ampicillin & 9 & $9(100)$ & 11 & $11(100)$ & 20 & $20(100)$ \\
\hline Amoxicillin-clavulanic acid & 9 & $8(88.9)$ & 11 & $11(100)$ & 20 & $19(95)$ \\
\hline Piperacillin & 9 & $9(100)$ & 11 & $11(100)$ & 20 & $20(100)$ \\
\hline Cefotaxime & 9 & $8(88.9)$ & 11 & $11(100)$ & 20 & $19(95)$ \\
\hline Ceftriaxone & 9 & $8(88.9)$ & 11 & $11(100)$ & 20 & $19(95)$ \\
\hline Ceftazidime & 9 & $7(77.8)$ & 11 & $7(63.6)$ & 20 & $14(70)$ \\
\hline Ciprofloxacin & 9 & $4(44.5)$ & 11 & $4(36.4)$ & 20 & $8(40)$ \\
\hline Gentamicin & 9 & $8(88.9)$ & 11 & $6(54.5)$ & 20 & $14(70)$ \\
\hline Meropenem & 9 & $3(33.3)$ & 11 & $5(45.5)$ & 20 & $8(40)$ \\
\hline Tetracycline & 9 & $7(77.8)$ & 11 & $10(90.9)$ & 20 & $17(85)$ \\
\hline Sulphamethoxazole - Trimethoprim & 9 & $6(66.7)$ & 11 & $11(100)$ & 20 & $17(85)$ \\
\hline Total & 99 & $77(77.8)$ & 121 & 98 (80.99) & 220 & $175(79.5)$ \\
\hline
\end{tabular}

\#T: Number of isolates tested, R\%: Percentage of resistant isolates 
care professionals, multiple invasive device and crosscontamination among patient's procedures.

In this study, the proportion of nosocomial MDR $A$. baumannii and $P$. aeruginosa surgical site infection (6.3\%) was comparable with previous reports from other part of Ethiopia (6.6\%) [25] and Ghana (8.5\%) [32]. However, it was lower than studies from Southeast China (28.5\%) [35]. This could be the difference in the type of surgery, handling of surgical equipments and age of study participants as the present study included patients of any age groups.

The proportion of nosocomial urinary tract infection linked with MDR $A$. baumannii and $P$. aeruginosa $(8.3 \%)$ isolates in the present study was consistent with a study conducted in Kenya (9\%) [36]. However, it was lower than studies in Gabon (52.8\%) [29], Indonesia (16.5\%) [31] and United States of America (USA) (16\%) [37]. This could be due to differences among study participants, catheterization and hospitalization.

The prevailing proportion of nosocomial BSI in this study (8.9\%) was comparable with studies done in USA (10\%) [37]. However, it was higher than studies from Indonesia (3.5\%) [31]. In contrast, lower findings were documented elsewhere in Africa $(20-70.3 \%)[29,38]$ and Southeast China (46.1\%) [35]. The observed difference might be due to non-sustainable infection control practices in hospitals, difference in use of invasive medical devices, procedures, hospital type and diverse nature of study participants.

The proportion of nosocomial MDR A. baumannii infection $(3.8 \%)$ in the present study was in agreement with earlier studies conducted in Africa (2.4-5.7\%) [26, $29,32]$. However, it was lower compared with findings from Sodo (15.3\%), Ethiopia [18] and Gaza city (6.9\%) [28]. In contrast, it was higher than findings from previous studies in elsewhere $(0.42-0.95 \%)[27,30]$. Similarly, the magnitude of nosocomial MDR $P$. aeruginosa infection (4.6\%) in the present study was comparable with a study in Uganda (5\%) [26]. However, it was lower than findings from other parts of Ethiopia (11.1-66.7\%) [8, 18, 25], Ghana (19.5\%) [32], India (76.8\%) [39] and Italy (8.7\%) [27]. In contrast, it was higher compared to a study in China $(0.36 \%)$ [30]. The variations might link with host, microbial and environmental factors.

In this study all isolates of MDR $P$. aeruginosa were resistant for ampicillin and amoxacillin-clavulanic acid. This was consistent with reports from Tikur Anbessa hospital, Ethiopia (25) and Southeast China [35] where 87.5 and $100 \%$ resistance rate against ampicillin and amoxacillin-clavulanic acid respectively were noticed. Moreover, all isolates of $P$. aeruginosa in the present study were resistant against piperacillin. This was significantly higher than studies from Italy (25\%) [27], Southeast China (12\%) [35], Turkey (28.7\%) [40] and Taiwan
(66.8\%) [41]. This might be associated with differences in the number of MDR strains of $P$. aeruginosa and patient type. The frustrating level of resistance against piperacillin antibiotic is an alarm for treatment to be guided with antimicrobial susceptibility testing as it was not prescribed in the study area (FHRH).

In this study, high levels of resistance to cephalosporins (cefotaxime $(63.6 \%)$ and ceftazidime $(100 \%)$ ) were obtained against $P$. aeruginosa isolates. This was coherent with studies in Uganda [23], India [39] and Taiwan [41] where $71-77 \%$ resistance against ceftazidime reported. Moreover, 70.8 and $92.8 \%$ level of resistance against cefotaxime were documented in Sodo, Ethiopia [18] and Southeast China [35], respectively. On the other hand, low level of resistance against ceftazidime reported in other parts of Ethiopia (12.5-29.1\%) [18, 25] and Italy (31\%) [27]. The highest level of resistance against third generation cephalosporins might be linked with excessive, mis and inappropriate use of these antibiotics in the area that drives selective pressure and emergence of MDR.

The resistance rate of $P$. aeruginosa isolates against meropenem $(45.5 \%)$ in the present study was coherent with other studies in Ethiopia (41.7\%) [18] and Asia $(36.6-54 \%)[33,39]$. However, higher level of resistance against meropenem was documented in Taiwan (73.2\%) [41] and Saudi (81.8\%) [42]. The relatively lower proportion resistance against meropenem in the present study might be linked with the nonexistence of meropenem prescription practice in the study hospital. In contrast, lower level of resistance against meropenem was reported in Uganda (14\%) [26] and Turkey (20.4\%) [40]. This could be due to variation in the availability of meropenem in each localities, prescription difference, misuse and inappropriate use of antibiotics.

In this study, all isolates of MDR A. baumannii were resistance against ampicillin. This was parallel with studies conducted in Tikur Anbessa Hospital (88.2\%), Ethiopia [25] and Southeast China (100\%) [35]. Moreover, high level (88.9\%) of MDR A. baumannii isolates resistance to amoxacillin clavulanic acid in the present study was comparable with earlier studies in Nigeria [43] and Southeast China [35], where all isolates of A. baumannii were resistant against amoxacillin- clavulanic acid.

All isolates of $A$. baumannii were resistant to piperacillin in the present study. This was comparable with studies conducted in Southeast Asia (83.7\%) [35] and Italy (81) [27]. Similarly, A. baumannii isolates also revealed 77.8 and $88.9 \%$ resistance against ceftazidime and cefotaxime, respectively which was comparable with studies done in Tanzania [44], Southeast China [35] and Italy [27], where resistance against ceftazidime and cefotaxime reported in $71-97.1 \%$ and $54.4-100 \%$ of isolates, 
respectively. Furthermore, $33.3 \%$ of $A$. baumannii isolates from the present findings showed resistance against meropenem. The finding was relatively similar with studies conducted in Sodo, Ethiopia [18], Tanzania [44] and Nigeria [43] where, $30.2-40 \%$ resistance rate against meropenem reported. However, in Saudi [42], resistance against meropenem was reported in $90.5 \%$ of A. baumannii isolates, respectively.

The resistance level of MDR isolates of $A$. baumannii to ciprofloxacin $(44.5 \%)$ in the present study was lower than studies done in Tikur Anbessa Hospital (70.6\%) and Sodo (88.4\%), Ethiopia [18, 25], Uganda (78\%) [26], Nigeria (100\%) [43], Southeast China (89.6\%) [35] and Italy (84\%) [27]. The low resistance rate against ciprofloxacin and meropenem in the present study might be due to the high price and unavailability of the drugs in the hospital.

In this study, all isolates of $A$. baumannii and $P$. aeruginosa were MDR (100\%). This finding was consistent with studies conducted in Sodo (81.4 \& 83.3\%), Ethiopia [18], Tanzania (100\%) [44], Ghana (100\%) [30] and India (76.8\%) [43]. However, it was higher than reports from Uganda (38 \& 40\%) [26], Saudi (28.2\%) [42], Saudi Arabia (71.8\%) [45] and Italy (20 \& 54\%) [27]. Therefore, this very alarming MDR proportion of $A$. baumannii and $P$. aeruginosa isolates in the study area needs urgent intervention and strict adherence to infection control practices to contain them.

The high MDR proportion observed in two nonfermenter gram negative bacilli in this study is probably related to the contaminations and cross transmission of these bacteria from hospital environment [18], hands of healthcare workers, frequent use of broad spectrum antibiotics, inherent resistance nature to many antimicrobial agents and the ability of pathogens persist in the environment, and on medical devices for a longer time [2]. Moreover, high prescription practice of common antibiotics including third generation cephalosporins and use of drugs outside the hospital might contribute for the high resistance rate of $A$. baumannii and $P$. aeruginosa to different classes of antibiotics.

This study was limited to participants admitted in hospital but nosocomial infection that arose after discharge was not detected. The single-centre nature of the study introduced local practice bias. The study also unable to demonstrate cross-transmission of pathogens and ignored the effect of duration of antibiotic therapy at individual patient level. Moreover, certain potential confounders could not be included in the analysis.

\section{Conclusions}

Alarming proportion of nosocomial MDR A. baumannii and $P$. aeruginosa infection obtained in the study area.
All $A$. baumannii and $P$. aeruginosa isolates were resistant for at least three antibiotics representative of different classes. Therefore, urgent intervention towards nosocomial infection prevention practices required and treatment of patients on care should be guided with antimicrobial susceptibility testing.

\section{Abbreviations \\ AMC: Amoxicillin-clavulanic acid; AMP: Ampicillin; AMR: Antimicrobial resistance; APHI: Amhara Public Health Institute; ATCC: American Type Culture Collection; BA: Blood agar; BSI: Blood Stream Infection; CAZ: Ceftazidime; CFU: Colony Forming Unit; CIP: Ciprofloxacin; CLSI: Clinical and Laboratory Standards Institute; CN: Gentamicin; ESKAPE: (Enterococcus faecium, Staphylococcus aureus, Klebsiella pneumoniae, Acinetobacter baumannii, Pseudomonas aeruginosa, Enterobacter species); FHRH: Felegehiwot Referral Hospital; ICU: Intensive Care Unit; MAC: MacConkey agar; MDR: Multi-Drug Resistance; MEM: Meropenem; MHA: Mueller- Hinton agar; ml: Milliliters; NI: Nosocomial Infection; PIP: Piperacillin; SOPS: Standard Operating Procedures; SSI: Surgical Site Infection; SXT: Sulfamethoxazole -Trimethoprim; UK: United Kingdom; USA: United States of America; UTI: Urinary Tract Infection; WHO: World Health Organization}

\section{Acknowledgements}

We would like to acknowledge College of Medicine and Health Sciences, Bahir Dar University for their assistance to conduct this study. We would like to acknowledge Felegehiwot Referral Hospital and Amhara Public Health Institute for their permission to carry out the laboratory work in their setting. We also acknowledge the technical support of the staff of $\mathrm{FHRH}$ and $\mathrm{APHI}$. We also acknowledge Amhara Regional Health Bureau for their permission to conduct the study in FHRH. We are also grateful to all patients involved in this study.

\section{Authors' contributions}

HM: Conceptualized and designed the study, performed the laboratory investigation. Collected the data, analyzed the data and drafted the manuscript. FB: Conceptualized the study, supervised the data collection and revised the manuscript. All authors have read and approved the manuscript, and ensure that this is the case. WM: Conceptualized and designed the study, supervised the laboratory investigation and data collection, analyzed the data, wrote, revised and critically edited the manuscript and significantly contributed to the scientific content of the study.

\section{Funding}

The research project was financed by College of Medicine and Health Sciences of Bahir Dar University. The fund was utilized for purchasing reagents, data collection, analysis and interpretation.

\section{Availability of data and materials}

The finding of this study is generated from the data collected and analyzed based on the stated methods and materials. All the data are already found in the manuscript and there are no supplementary flies. The original data supporting this finding will be available at any time upon request.

\section{Ethics approval and consent to participate}

Ethical clearance was secured from Institutional Review Board of College of Medicine and Health Sciences, Bahir Dar University. Permission letter was obtained from the Amhara Public Health Institute and FHRH prior to data collection. All the study participants were informed about the purpose of the study. Written informed consent was obtained from each study participants and guardian for participants under 16 years old before clinical examination and sample collection. Information obtained in the course of the study was kept confidential. Participants who were positive for the pathogen reported to physicians for treatment and any other care.

\section{Consent for publication}

Not applicable. 
Received: 30 September 2019 Accepted: 21 January 2020 Published online: 30 January 2020

\section{References}

1. Poza M, Gayoso C, Gómez MJ, Rumbo-Feal S, Tomás M, Aranda J, et al. Exploring bacterial diversity in hospital environments by GS-FLX titanium pyrosequencing. PLoS One. 2012;7(8):e44105.

2. Exner M, Bhattacharya S, Christiansen B, Gebel J, Goroncy-Bermes P, Hartemann $P$, et al. Antibiotic resistance: what is so special about multidrugresistant gram-negative bacteria? GMS Hyg Infect Control. 2017;12:1-24.

3. Pritsch M, Zeynudin A, Messerer M, Baumer S, Liegl G, Schubert S, et al. First report on bla $a_{\mathrm{NDM}-1}$-producing Acinetobacter baumannii in three clinical isolates from Ethiopia. BMC Infect Dis. 2017;17:180. https://doi.org/. https:// doi.org/10.1186/s12879-017-2289-9.

4. Revelas A. Healthcare-associated infections: a public health problem. Niger Med J. 2013;53(2):59-64.

5. Mitchell BG, Shaban RZ, Macbeth D, Wood CJ, Russo PL. The burden of healthcare-associated infection in Australian hospitals: A systematic review of the literature. Infect Dis Health. 2017;22:117-28.

6. World Health Organization. Report on the burden of endemic health careassociated infection worldwide. WHO. 2014.

7. Percival SL, Suleman L, Vuotto C, Donelli G. Healthcare-associated infections, medical devices and biofilms: risk, tolerance and control. J Med Microbiol. 2015;64:323-34.

8. Tolera M, Abate D, Dheresa, M, Marami D. Bacterial nosocomial infections and antimicrobial susceptibility pattern among patients admitted at Hiwot Fana Specialized University hospital, East Ethiop Adv Med 2018; 2018 : https://doi.org/https://doi.org/10.1155/2018/2127814.

9. Bekele T, Tesfaye A, Sewunet T, Waktola HD. Pseudomonas aeruginosa isolates and their antimicrobial susceptibility pattern among catheterized patients at Jimma University Teaching Hospital, Jimma, Ethiopia. BMC Res Notes. 2015;8:488. https://doi.org/10.1186/s13104-015-1497-x.

10. European Centre for Disease Prevention and Control. Point prevalence survey of health care associated infections and antimicrobial use in European acute care hospitals - protocol version 5.3. Stockholm: ECDC; 2016.

11. Morales E, Cots F, Sala M, Comas M, Belvis F, Riu M, et al. Hospital costs of nosocomial multi-drug resistant Pseudomonas aeruginosa acquisition. BMC Health Serv Res. 2012;12:122. https://doi.org/. https://doi.org/10.1186/1472 6963-12-122.

12. Huang $\mathrm{H}$, Chen B, Liu G, Ran J, Lian X, Huang X, et al. A multi-center study on the risk factors of infection caused by multi-drug resistant Acinetobacter baumannii. BMC Infect Dis. 2018;18:11. https://doi.org/. https://doi.org/10. 1186/s12879-017-2932-5.

13. World Health Organization. Guidelines for the prevention and control of carbapenem-resistant Enterobacteriaceae, Acinetobacter baumannii and Pseudomonas aeruginosa in health care facilities. Geneva. WHO. 2017.

14. World Health Organization. Global priority list of antibiotic-resistant bacteria to guide research, discovery, and development of new antibiotics. WHO. 2017.

15. Santajit $\mathrm{S}$, Indrawattana N. Mechanisms of antimicrobial resistance in ESKAPE pathogens. Biomed Res Int. 2016;2016:2475067. https://doi.org/. https://doi.org/10.1155/2016/2475067.

16. Ali J, Rafiq QA, Ratcliffe E. Antimicrobial resistance mechanisms and potential synthetic treatments. Future Sci OA. 2018;4(4):FSO290.

17. Bassetti M, Vena A, Croxatto A, Righi E, Guery B. How to manage Pseudomonas aeruginosa infections. Drugs Context. 2018;7:212527. https:// doi.org/. https://doi.org/10.7573/dic.212527.

18. Solomon FB, Wadilo F, Tufa EG, Mitiku M. Extended spectrum and metalo beta-lactamase producing airborne Pseudomonas aeruginosa and Acinetobacter baumannii in restricted settings of a referral hospital: a neglected condition. Antimicrob Resist Infect Control. 2017;6:106. https:// doi.org/. https://doi.org/10.1186/s13756-017-0266.

19. Manenzhe RI, Zar HJ, Nicol MP, Kaba M. The spread of carbapenemaseproducing bacteria in Africa: a systematic review. Antimicrob Chemother. 2015;70(1):23-40. https://doi.org/. https://doi.org/10.1093/jac/dku356.

20. Rods GP. UK standards for microbiology investigations. Public Health England. 2014

21. Cheesbourgh M District laboratory practice in tropical countries. Part II. $2^{\text {nd }}$ ed. NewYork Cambridge University Press; 2006.
22. Bauer AW, Kirby WMM, Sherris JC, Turck M. Antibiotic susceptibility testing by standard single disc method. Am J Clin Path. 1966;45:493-6.

23. Clinical Laboratory Standards Institute. Performance Standards for Antimicrobial Susceptibility Testing; Twenty-Fifth Informational.Supplement. CLSIdocumentM100-S25.Wayne, PA:CLSI, 2017.

24. Johnson AP. Surveillance of antibiotic resistance. Philos Trans R Soc Lond Ser B Biol Sci. 2015;370(1670):20140080.

25. Asres GS, Legese MH, Woldearegay GM. Prevalence of multidrug resistant bacteria in postoperative wound infections at Tikur Anbessa specialized hospital, Addis Ababa. Ethiopia Arch Med. 2017;9(4):1-9.

26. Kateete DP, Nakanjako R, Okee M, Joloba ML, Najjuka CF. Genotypic diversity among multidrug resistant Pseudomonas aeruginosa and Acinetobacter species at Mulago Hospital in Kampala. Uganda BMC Res Notes. 2017;10:284. https://doi.org/. https://doi.org/10.1186/ s13104-017-2612-y.

27. De Francesco MA, Ravizzola G, Peroni L, Bonfanti C, Manca N. Prevalence of multidrug-resistant Acinetobacter baumannii and Pseudomonas aeruginosa in an Italian hospital. J Infect Public Health. 2013;6:179-85.

28. Al Jarousha AM, ElJadba AHN, AIAfifi AS, ElQouqa IA. Nosocomial multidrug resistant Acinetobacter baumannii in the neonatal intensive care unit in Gaza City. Palestine Int J Infect Dis. 2009;13:623-8.

29. Scherbaum M, Kösters K, Mürbeth RE, Ngoa UA, Kremsner PG, Lell B, et al. Incidence, pathogens and resistance patterns of nosocomial infections at a rural hospital in Gabon. BMC Infect Dis. 2014;14:124.

30. $X u$ H, Gang L, Li Y, Min L, Jing W. The epidemiology of multidrug-resistant bacteria colonization and analysis of its risk factors in intensive care unit. Chin Crit Care Med. 2015;27(8). https://doi.org/). https://doi.org/10.3760/cma. j.issn.2095-4352.2015.08.01

31. Murni IK, Duke T, Daley AJ, Kinney S, Soenarto Y. Antibiotic resistance and mortality in children with nosocomial blood stream infection in a teaching hospital in Indonesia. Southeast Asian J Trop Med Public Health. 2016;47(5): 983-93.

32. Agyepong N, Govinden U, Owusu-Ofori A, Essack SY. Multidrug-resistant gram-negative bacterial infections in a teaching hospital in Ghana. Antimicrob Resist Infect Control 2018; 7:37. https://doi.org/https://doi.org/10. $1186 /$ s13756-018-0324-2

33. Shanthi M, Sekar U. Multi-drug resistant Pseudomonas aeruginosa and Acinetobacter baumannii infections among hospitalized patients: risk factors and outcomes. JAPI. 2009;57:636-40.

34. Raman G, Avendano EE, Chan J, Merchant S, Puzniak L. Risk factors for hospitalized patients with resistant or multidrug-resistant Pseudomonas aeruginosa infections: a systematic review and meta-analysis. Antimicrob Resist Infect Control 2018; 7:79. https://doi.org/https://doi.org/10.1186/ s13756-018-0370-9.

35. Li L, Dai J, Xu L, Chen Z, Li X, Liu M, et al. Antimicrobial resistance and pathogen distribution in hospitalized burn patients a multicenter study in Southeast China. Med. 2018:97(34):1-9.

36. Jiménez-Guerra G, Heras-Cañas V, Gutierrez-Soto M, Del Pillar A-PM, Expósito-Ruiz M, Navarro-Marí JM, et al. Urinary tract infection by Acinetobacter baumannii and Pseudomonas aeruginosa: evolution of antimicrobial resistance and therapeutic alternatives. J Med Microbiol. 2018; 67:790-7.

37. Dent LL, Marshall DR, Pratap S, Hulette RB. Research article multidrug resistant Acinetobacter baumannii: a descriptive study in a city hospital. BMC Infect Dis. 2010;10:196.

38. Saïdani M, Boutiba I, Ghozzi R, Kammoun A, Redjeb SB. Bacteriologic profile of bacteremia due to multi-drug resistant bacteria at Charles-Nicolle hospital of Tunis. Med Mal Infect. 2006;36:163-6.

39. Bhatt P, Rathi KR, Hazra S, Sharma A, Shete V. Prevalence of multidrug resistant Pseudomonas aeruginosa infection in burn patients at a tertiary care Centre. Indian J Burn. 2015;23(1):56-9.

40. Savap L, Duran N, Savap N, Nlen Y, Ocak S. The prevalence and resistance patterns of Pseudomonas aeruginosa in intensive care units in a university hospital. Turk J Med Sci. 2005:35:317-22.

41. Hsueh P, Liu Y, Yang D, Yan J, Wu T, Huang W, et al. Multicenter surveillance of antimicrobial resistance of major bacterial pathogens in intensive care units in 2000 in Taiwan. Microb Drug Resist. 2001;7(4):373-82.

42. Somily AM, Absar MM, Arshad MZ, Aska A, Shakoor ZA, Fatani AJ, et al. Antimicrobial susceptibility patterns of multidrug resistant Pseudomonas aeruginosa and Acinetobacter baumannii against carbapenems, colistin, and tigecycline. Saudi Med J. 2012;33(7):750-5. 
43. Nwadike UV, Ojide KC, Kalu IE. Multidrug resistant Acinetobacter infection and their antimicrobial susceptibility pattern in a Nigerian tertiary hospital icu. Afr J Infect Dis. 2014;8(1):14-8.

44. Manyahi J, Matee M, Majigo M, Moyo S, Mshana SE, Lyamuya EF.

Predominance of multi-drug resistant bacteria pathogens causing surgical site infections in Muhimbili national hospital. Tanzania BMC Res Notes. 2014;7:500.

45. Ibrahim ME. Prevalence of Acinetobacter baumannii in Saudi Arabia: risk factors, antimicrobial resistance patterns and mechanisms of carbapenem resistance. Ann Clin Microbiol Antimicrob. 2019;18(1):2-12.

\section{Publisher's Note}

Springer Nature remains neutral with regard to jurisdictional claims in published maps and institutional affiliations.

Ready to submit your research? Choose BMC and benefit from:

- fast, convenient online submission

- thorough peer review by experienced researchers in your field

- rapid publication on acceptance

- support for research data, including large and complex data types

- gold Open Access which fosters wider collaboration and increased citations

- maximum visibility for your research: over $100 \mathrm{M}$ website views per year

At $\mathrm{BMC}$, research is always in progress.

Learn more biomedcentral.com/submissions 